\title{
Discussion on the Management of Safe Medication in the Treatment of Internal Diseases of Traditional Chinese Medicine
}

\author{
Xuan Gong1,2, Liming Zhang1, Lizhi Niu ${ }^{2 *}$ \\ ${ }^{1}$ Beijing University of Chinese Medicine, Beijing 100029, China \\ ${ }^{2}$ Chifeng Municipal Hospital, Chifeng 024000, Inner Mongolia, China \\ *Corresponding author: Lizhi Niu, 18811132508@163.com
}

\begin{abstract}
Objective: To discuss the management of safe medication in the treatment of internal diseases in traditional Chinese medicine. Methods: 124 patients with medical diseases were divided into experimental group and control group by random number table method, with 62 cases in each group. The experimental group implemented safe medication management, and the control group implemented routine medication management, all of which were treated for 4 consecutive weeks. The comparison of the improvement of the quality of life, the compliance rate of safe medication and the adverse drug reactions of the two groups after clinical treatment were compared. Results: After 8 weeks of treatment, there was a significant difference in clinical efficacy between the two groups of patients. In terms of quality-of-life scores, the scores of the experimental group were better than those of the control group. The living ability, condition, physical strength and other conditions of patients were significantly different. The t-values were 15.3427, 14.0275, and 7.7113, respectively, and $\mathrm{P}<0.01$ was statistically significant. The compliance rates of patients in the experimental group and observation group were $95.16 \%$ and $70.96 \%$, respectively, with significant differences $(\mathrm{P}<0.05)$. In terms of the incidence of adverse drug events, the experimental group was $3.22 \%$, which was significantly lower than the observation group's $8.06 \%(\mathrm{P}<0.05)$. Conclusion: The implementation of safe medication management in the clinical treatment of TCM internal diseases can improve the quality of life of patients to a certain extent, increase their safe medication compliance rate, and reduce the incidence of adverse medication events. The clinical application value is extremely high, and it can be widely promoted and applied.
\end{abstract}

Keywords: Chinese medicine; Internal medicine; Disease treatment; Safe medication management

Publication date: July 2021; Online publication: July 31, 2021

\section{Introduction}

Chronic kidney disease and heart disease are common diseases in the internal medicine department of the Chinese Medicine Hospital. If the condition is not well controlled, it can easily progress to major diseases such as nephritis, kidney failure, and cardiac dysfunction, which will affect the long-term quality of life. In internal medicine, the treatment cycle is relatively long, and it is very difficult for patients in the later stage of treatment. Due to the phenomenon of misuse of drugs and indiscriminate use of drugs, many adverse reactions occur, leading to further development and deterioration of the disease, making the treatment more difficult.

With the increase in the types of medical diseases, the difficulty of treatment has further increased. In order to effectively improve the cure rate, standardization of scientific drug safety management must be carried out during the treatment process to reduce the risk of related complication $\mathrm{s}^{[1]}$. In order to explore the effect of safe medication management in the clinical treatment of TCM gastroenterology patients, 
through the management of 100 selected patients who met the research conditions among the 7920 patients with medical diseases admitted to their department in 2018. Experimental research has found that after the implementation of safe medication management, the quality of important talents can be well controlled, and the information-based medication process has been gradually formed; avoiding the occurrence of misuse of medications and indiscriminate medications, as well as reducing the contradiction between doctors and patients. Before and after the implementation of safe medication management, there were significant differences in various clinical indicators of patients. Among them, the most typical was the incidence of adverse events of medication.

After the implementation of safe medication management, the incidence of adverse events was significantly lower than before the implementation $(\mathrm{P}<0.05)$. Liu Yanru ${ }^{[2]}$ hopes to find out the effective treatment of scientific medication methods for patients with internal diseases through the research on the implementation of safety management in the treatment of internal medicine diseases in traditional Chinese medicine (TCM). Through clinical trial research, it is found that after the implementation of safe medication management for patients in the selected study group of the 63 patients, only 4 had adverse reactions, while another group of 63 patients who did not implement safe medication management had 9 adverse reactions, indicating that safe medication management can effectively control the incidence of adverse drug reactions and treat patients clinically. Thus, it has a positive role in promoting. The author of this article combines the research materials of medical experts and hopes to explore the clinical application value of safe medication management for the treatment of internal medicine diseases through clinical trial research, and provide guidance for the realization of scientific treatment of internal diseases.

\section{Materials and methods}

\subsection{General information}

The 124 internal medicine patients admitted to our hospital from February 2018 to February 2019 were selected for research. The research has been approved by the ethics committee of our hospital.

\subsubsection{Diagnosis and selection criteria}

(1) Comply with the diagnostic criteria of nephrology and cardiology in the "Guidelines for Diagnosis and Prevention of Traditional Chinese Medicine Internal Medicine," with no abnormal imaging diagnosis.

(2) Long-term drug treatment has no obvious effect.

(3) Age of the patients must be within 24-62 years old.

(4) Able Barrier-free communication with medical staff.

(5) The patient's family members have signed the informed consent form for the study.

\subsubsection{Exclusion criteria}

(1) Does not meet the diagnostic criteria of TCM internal medicine.

(2) Have a history of severe drug allergy.

(3) Congenital heart disease and renal insufficiency.

(4) Withdraw from the study halfway and did not complete the follow-up.

100 cases of TCM internal medicine patients were divided into experimental group and control group by random number table method, with 62 cases in each group. There was no statistically significant difference in general information between the two groups $(\mathrm{P}>0.05)$, see Table 1.

\subsection{Method}

The control group implements routine medication management. The experimental group implemented safe medication management. 
Table 1. Comparison of general information between the two groups of patients [( $\overline{\mathrm{x}} \pm \mathrm{s}), \mathrm{n}(\%)]$

\begin{tabular}{|c|c|c|}
\hline Group & & Control group \\
\hline Number of cases & & 62 \\
\hline $\begin{array}{l}\text { Average age (One } \\
\text { year old) }\end{array}$ & & $46.4 \pm 5.6$ \\
\hline Average weight (kg) & & $51.4 \pm 5.2$ \\
\hline Inclusion criteria & $\begin{array}{l}(1) \\
(2) \\
(3) \\
(4) \\
(5)\end{array}$ & $\begin{array}{l}\text { Comply with the "Guidelines for Diagnosis and Prevention of } \\
\text { Traditional Chinese Medicine Internal Medicine" in the diagnosis } \\
\text { standards of nephrology and cardiology diseases, with no abnormal } \\
\text { imaging diagnosis. } \\
\text { No obvious effect after long-term drug treatment. } \\
\text { 24-62 years old. } \\
\text { barrier-free with medical staff Communication. } \\
\text { The patient's family members have signed the informed consent form for } \\
\text { the study. }\end{array}$ \\
\hline
\end{tabular}

(1) Does not meet the diagnostic criteria of TCM internal medicine.

Exclusion criteria

(2) Have a history of severe drug allergy.

(3) Congenital heart disease and renal insufficiency.

(4) Withdraw from the study halfway and did not complete the follow-up.

\subsubsection{Disease knowledge awareness}

After the patients received treatment, the responsible physicians and nurses explain the knowledge of disease treatment to the patients, and distribute the medication safety knowledge manual, to enhance the patients' awareness of medication safety and understand the matters that should be paid attention to in the medication process.

\subsubsection{Explanation of common information of the medication.}

Explained to the patients about the dosage and regularity of the medication, also the possible complications and adverse reactions, as well as introduced them in advance the matters that should be paid attention to during the medication process. At the same time, with the help of not taking medication on time, taking medication not according to doctor's advice or taking irregularly will result in real cases of serious consequences caused by drugs will let patients understand the importance of safe medication.

\subsubsection{Efficacy records}

Regularly record the infiltration recovery of patients after medication, and report to the attending physician, so that the physician can make reasonable medication adjustments.

\subsubsection{Intimate service of medication for elderly patients}

As elderly patients have poor physical function indicators, it is easy to forget to take or miss medication, remind family members to guide elderly patients to take medication on time, and to understand the patient's medication status through telephone follow-up and WeChat follow-up, and give it based on the feedback of patients and family members Scientific medication recommendations.

\subsection{Observation indicators and evaluation of curative effect}

After treatment for a period of time, referring to the literature evaluation, markedly the effectiveness, after 
4 weeks of medication, the patient's quality of life has been significantly improved, the condition has been significantly improved, the living ability has been significantly improved, the physical recovery status is good, the rate of safe medication compliance and poor medication ${ }^{[3-5]}$. The reaction and other conditions have been significantly improved. Effectiveness, after 4 weeks of medication, the condition is relieved and the sleep state is good. The invalidness is that there are no effects after 8 weeks of treatment. "Total effective rate = apparent efficiency + effective rate."

\subsection{Statistical methods}

Statistical analysis was performed with SPSS18.0 software. Measurement data was expressed by independent sample t-test for comparison between groups, paired sample t-test was used for comparison between groups, count data was expressed by rate, and comparison is expressed by $\mathrm{X}^{2}$ test. $\mathrm{P}<0.05$ indicates that the difference is statistically significant.

\section{Results}

\subsection{Comparison of clinical efficacy between the two groups of patients}

The quality-of-life scores of patients in the control group and the test group were significantly different. The effective rates of treatment were $88.70 \%$ and $95.16 \%$, respectively. There were significant differences in the living ability, condition, and physical strength of the patients. The $t$ values were 15.3427, 14.0275, and 7.7113, respectively, $\mathrm{P}<0.01$ statistics It is meaningful to learn, see Table 2.

Table 2. Comparison of the quality-of-life scores of the two groups of patients ( $\bar{x} \pm s$, points )

\begin{tabular}{ccccc}
\hline Group & Viability & State of an illness & Physical power & Effective rate \\
\hline Test group $(\mathrm{n}=62)$ & $15.98 \pm 2.11$ & $12.98 \pm 1.12$ & $6.88 \pm 2.36$ & $59(95.16 \%)$ \\
Control group & $11.12 \pm 1.33$ & $15.97 \pm 1.25$ & $4.26 \pm 1.26$ & $55(88.70 \%)$ \\
$(\mathrm{n}=62)$ & 15.3427 & 14.0275 & 7.7113 & - \\
$\mathrm{t}$ & 0.000 & 0.000 & 0.000 & - \\
$\mathrm{P}$ & & & & \\
\hline
\end{tabular}

\subsection{Comparison of the compliance rate of safe medication between the two groups}

Compared with the control group, the experimental group has a significantly higher compliance rate of patients with safe medication, and the difference between the two was significant $(\mathrm{P}<0.05)$, see Table 3.

Table 3. Comparison of the safety medication compliance of the two groups of patients [n (\%)]

\begin{tabular}{ccccc}
\hline Group & Good & Average & Poor & Compliance Rate \\
\hline $\begin{array}{c}\text { Test group }(\mathrm{n}=62) \\
\text { Control group }\end{array}$ & $19(30.64)$ & $25(40.32)$ & $18(9.03)$ & $44(70.96 \%)$ \\
$(\mathrm{n}=62)$ & $48(77.41)$ & $11(17.74)$ & $3(4.83)$ & $59(95.16 \%)$ \\
$\mathrm{X}^{2}$ & & & & 21.8312 \\
$P$ & & & & 0.000 \\
\hline
\end{tabular}

\subsection{Comparison of the incidence of adverse drug events between the two groups}

The results showed that the incidence of adverse events in the experimental group with safe medication management was significantly lower than that in the control group with conventional medication 
management $(\mathrm{P}<0.05)$. See Table 4.

Table 4. Comparison of the incidence of adverse drug events between the two groups $(\mathrm{n}, \%)$

\begin{tabular}{ccccc}
\hline Group & Sick & Swirl & Diarrhea & Incidence of adverse events $(\boldsymbol{\%})$ \\
\hline Test group $(\mathrm{n}=62)$ & 2 & 2 & 1 & $5(8.06 \%)$ \\
Control group $(\mathrm{n}=62)$ & 1 & 1 & 0 & $2(3.22 \%)$ \\
$\mathrm{X}^{2}$ & & & 51.2712 \\
$P$ & & & 0.000 \\
\hline
\end{tabular}

\section{Discussion}

The results of this study show that the use of safe medication management to treat patients with TCM internal diseases has a significant effect. There was a significant difference in the quality of life scores between the control group and the test group. The effective rates of treatment were $88.70 \%$ and $95.16 \%$, respectively. There were significant differences in the living ability, condition, and physical strength of the patients. $\mathrm{T}$ values were $15.3427,14.0275,7.7113, \mathrm{P}<0.01$ Statistical significance; by comparing the two groups of safe medication compliance rate, the experimental group was significantly higher than the control group, $95.16 \%>70.96 \%,<0.01$ was statistically significant; in terms of the incidence of adverse drug events, only two cases of the experimental group had adverse events. The incidence rate was $3.22 \%$, while five cases occurred in the control group, the incidence rate was $8.06 \%$. Based on various clinical indicators, medication safety management has high clinical guidance value for promoting the recovery of patients, reducing adverse reactions, and reducing medication risks, and was suitable for effective treatment of TCM internal diseases. However, this study also has limitations, such as failure to continuously observe the longterm efficacy and too small sample size. In the future, higher quality studies will be carried out to continuously verify this conclusion.

\section{Disclosure statement}

The author declares no conflict of interest.

\section{References}

[1] Xi XX, 2020, Discussion on the Management of Safe Medication in the Treatment of Internal Diseases of Traditional Chinese Medicine, Journal of Traditional Chinese Medicine Management, (11):28.

[2] Liu YR, 2019, Discussion on the Management of Safe Medication in the Treatment of Internal Diseases of Traditional Chinese Medicine, China Health Industry, (11):15.

[3] Mao LY, Li J, Zhuang J, 2020, Management of Safe Medication in the Treatment of Diseases in the Department of Nephrology of Traditional Chinese Medicine, Chinese Medicine Management Journal, (11): 14 .

[4] Sun AZ, Jin LF, 2020, Clinical Analysis of the Safety Management of Nursing Medication in the Department of Cardiology, Diabetes World, 17(03):271.

[5] Zhang N, Ma Y, Wang R, et al., 2019, The Application of Closed-Loop Management in the Safe Medication of Elderly Pulmonary Tuberculosis Patients, Chinese Journal of National Defence Tuberculosis, 41(11):128-129. 